\title{
Light ions response of silicon carbide $\operatorname{detectors}^{1}$
}

\author{
M. De Napoli ${ }^{a}$, G. Raciti ${ }^{\mathrm{a}, *}$, E. Rapisarda $^{\mathrm{a}}$, C. Sfienti $^{\mathrm{b}}$ \\ ${ }^{a}$ Dipartimento di Fisica, Università degli studi di Catania and Sezione INFN \\ Via S.Sofia,64 I-95123 Catania-Italy \\ ${ }^{\mathrm{b}}$ GSI-Darmstadt-D-64291-Germany
}

\begin{abstract}
Silicon carbide (SiC) Schottky diodes $21 \mu \mathrm{m}$ thick with small surfaces and high N-dopant concentration have been used to detect alpha particles and low energy light ions. In particular ${ }^{12} \mathrm{C}$ and ${ }^{16} \mathrm{O}$ beams at incident energies between 5 and 18 $\mathrm{MeV}$ were used. The diode active-region depletion-thickness, the linearity of the response, energy resolution and signal rise-time were measured for different values of the applied reverse bias. Moreover the radiation damage on $\mathrm{SiC}$ diodes irradiated with $53 \mathrm{MeV}{ }^{16} \mathrm{O}$ beam has been explored. The data show that $\mathrm{SiC}$ material is radiation harder than silicon but at least one order of magnitude less hard than epitaxial silicon diodes. An inversion in the signal was found at a fluence of $10^{15}$ ions $/ \mathrm{cm}^{2}$.
\end{abstract}

Key words: SiC-Silicon Carbide, Semiconductors, Radiation Detectors, Radiation Damage

PACS: 29.40, 07.85.F, 07.77.K, 


\section{Introduction}

In the last years the use of electronic devices and sensors in very harsh environments at elevated temperatures, high-power, high-frequency and high radiation fields has became the subject of research and development in various fields. In particular the search for new materials, more suitable for such extreme operating conditions than the usual silicon semiconductor has received a lot of interest. Among the investigated materials, the silicon carbide ( $\mathrm{SiC})$ semiconductor has raised large interest and has been already used in a wide range of applications $[1,2,3,4,5,6,7,8,8$. In particular, recent work has been done on the development of $\mathrm{SiC}$ radiation detectors [9,10] and on the characterization of their performances. SiC detectors were used with excellent results as neutron [11] and X-ray detectors operating at high temperatures [12. The charged-particle response characteristics have been measured by irradiating the detectors with alpha particle sources at energies up to $5.48 \mathrm{MeV}$ [13]14,15], and radiation damage effects were investigated with $24 \mathrm{GeV}$ protons and gamma rays [16,17,18], $300 \mathrm{MeV} / \mathrm{c}$ pions [19], neutrons [20] and protons, alphas and ${ }^{12} \mathrm{C}$ beams [21] at fluences up to $10^{16}$ particles $/ \mathrm{cm}^{2}$ [20] obtaining promising results. Indeed one of the most appealing property of the $\mathrm{SiC}$, as well as of other wide bandgap materials such as GaAs and diamond, is their predicted radiation hardness with respect to Silicon. Moreover SiC diodes show low reverse current even at the very high electrical voltage applicable because

\footnotetext{
* Corresponding author: Tel:+ 39-095-542 286 FAX: + 39-095-378 5225 Email address: raciti@lns.infn.it (G. Raciti).

1 Partly supported by the European Community in the framework of the "DIRAC secondary-beams" project, contract N.515873 under the "Structuring the European Research Area" Specific Programme Research Infrastructures Action.
} 
of their higher breakdown voltage with respect to $\mathrm{Si}$. Both properties make $\mathrm{SiC}$ diodes suitable for the construction of specific detector arrays. The inner tracking detectors, typically used in the high-radiation environment of particle physics experiments, particle and ion detector arrays operating in satellites or in the very harsh environment of laboratories producing radioactive ionbeams would particularly benefit from the mentioned properties. In spite of the notable development in growing, processing and producing good-quality and low-defect $\mathrm{SiC}$ diodes, work to date on the use of such diodes as detectors of particles and ions has been very limited [13]14,15,19,21]. We have therefore studied the response signal of high-quality $4 \mathrm{H}-\mathrm{SiC}$ Schottky diode to ${ }^{12} \mathrm{C}$ and ${ }^{16} \mathrm{O}$ ions and to an alpha particles source at various incident energies, in order to investigate the use of $\mathrm{SiC}$ detectors also in nuclear physics applications. The main objectives of the present work are the characterization of the signal response in term of linearity, energy resolution and rise-time as a function of the applied reverse bias and the investigation of its degradation subsequent to irradiation with light ions.

\section{Experimental details}

The Schottky diodes were fabricated by epitaxy onto high-purity $4 \mathrm{H}-\mathrm{SiC} \mathrm{n}$ type substrate from the ETC-Catania [22]. Figure 1] shows the layout of the detector. The nominal $\mathrm{n}^{-}$epitaxial layer nitrogen dopant concentration and thickness were, respectively, $1.5 \times 10^{16} \mathrm{~N} \mathrm{~cm}^{-3}$ and $21 \mu \mathrm{m}$. The dopant concentration and thickness of the $\mathrm{n}^{+}$side were respectively $7 \times 10^{18} \mathrm{~N} \mathrm{~cm}^{-3}$ and 279 $\mu \mathrm{m}$. The net doping and the thickness have been measured [22], respectively, by C-V (Hg-probe) and FTR Biorad QS-500. The nominal defects mean value 


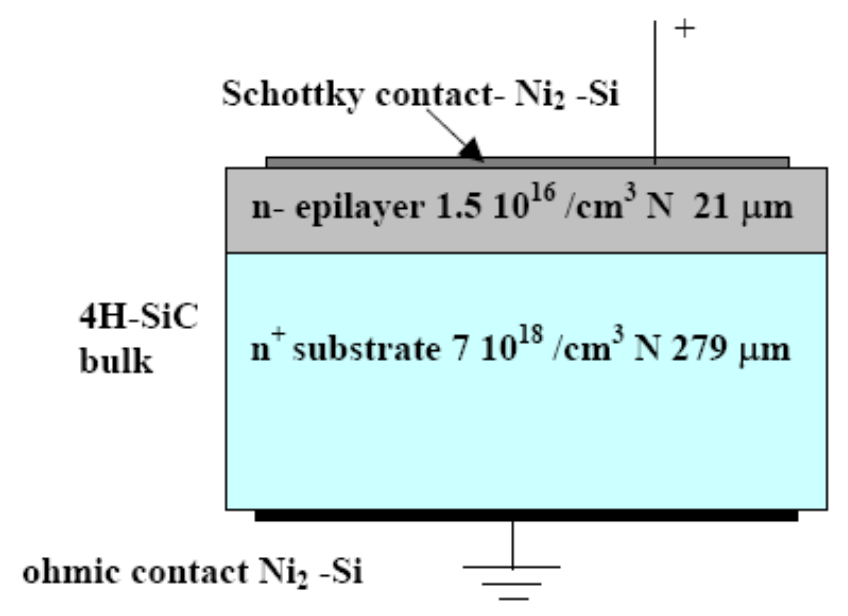

Fig. 1. The $4 \mathrm{H}-\mathrm{SiC}$ diode layout. The Schottky junction is realized by the $\mathrm{Ni}_{2} \mathrm{Si}$ layer, $0.2 \mu \mathrm{m}$ thick, deposited at $600^{\circ} \mathrm{C}$ on the front surface, the ohmic layer by $\mathrm{Ni}_{2} \mathrm{Si}$ deposited at $950^{\circ} \mathrm{C}$.

was $43 \mathrm{~cm}^{-3}$ as measured [22] by $\mu$ Raman spectroscopy with an Ar-laser at $514.5 \mathrm{~nm}$.

The Schottky junction was realized by a $0.2 \mu \mathrm{m}$ thick layer of $\mathrm{Ni}_{2} \mathrm{Si}$ deposited at $600{ }^{\circ} \mathrm{C}$ on the front surface, while the ohmic contact, on the back surface, was obtained with $\mathrm{Ni}_{2} \mathrm{Si}$ deposited at $950^{\circ} \mathrm{C}[23$.

The active areas of the different $\mathrm{SiC}$ detectors were of $0.5 \mathrm{x} 0.5,1 \mathrm{x} 1$ and $2 \mathrm{x} 2$ $\mathrm{mm}^{2}$ (see Fig. 2). The chips were glued on a brass foil $1 \mathrm{~mm}$ thick by a conductive glue and single contacts between the $\mathrm{Ni}_{2} \mathrm{Si}$ front surfaces and the pads of the board shown in Figure 2 were realized by $\mathrm{Al}$ wire $(2 \mu \mathrm{m}$ thick $)$ bonding.

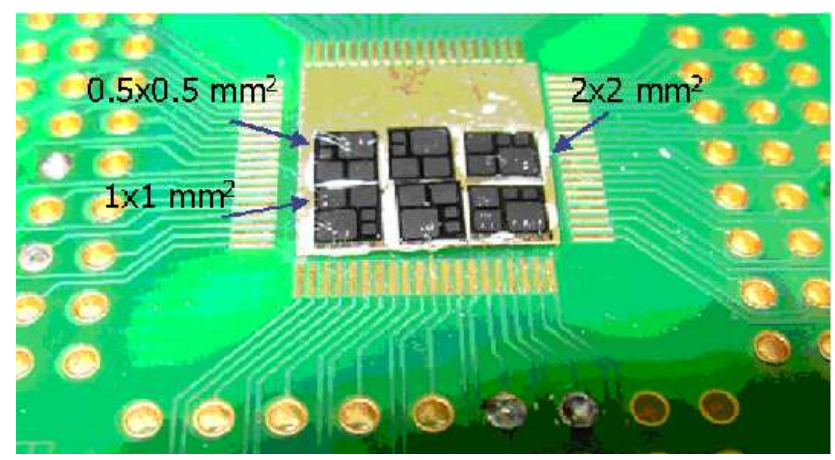

Fig. 2. Picture of the SiC detectors assembled on a board. 
Two boards with respectively six and five chips $2 \mathrm{x} 2 \mathrm{~mm}^{2}$ of surface were assembled, for a total number of eleven independent detectors. The third board was equipped with a Si detector $300 \mu \mathrm{m}$ thick and $3 \times 3 \mathrm{~cm}^{2}$ of surface which was used as a reference. The three boards were set-up in a scattering chamber at the Laboratori Nazionali del Sud (LNS-Catania) and operated under vacuum at $10^{-6}$ mbar. The active samples were irradiated with both $5.48 \mathrm{MeV}$ alpha particles from an ${ }^{241} \mathrm{Am}$ source and ${ }^{12} \mathrm{C}$ and ${ }^{16} \mathrm{O}$ beams accelerated by the LNS Tandem VdG at different energies and currents. The alpha source was set-up at a distance of $10 \mathrm{~cm}$ from the detector surface in order to get a near normal incidence of alpha particles on the detector. The ion beams were focused 60 $\mathrm{cm}$ downstream with respect to the detector position in order to achieve an uniform perpendicular irradiation in a spot of about $3 \mathrm{~mm}$ of diameter on the $\mathrm{SiC}$ surface. The boards holder was moved so as to center the detectors one by one. Standard electronics was used to process the signals: preamplifiers of $45 \mathrm{mV} / \mathrm{MeV}$ gain and amplifiers with $0.5 \mu$ sec shaping time. Data acquisition was based on CAMAC ADCs read out through a GPIB standard National Instruments interface and a data-acquisition program built in the LabView 7 framework. Moreover the preamplifier and amplifier signals were digitalized by a Tektronix TDS 5104B digital oscilloscope.

\section{Detector response}

It is well known that a reverse bias must be applied in order to create in the diode a depleted region which acts as the active region for the detection of the charges produced by ionization from the incoming charged particles.

The order of magnitude of this reverse bias could be a relevant parameter for 
specific applications. Therefore we measured at first the correlation between the thickness of the depleted layer and the applied bias, which is expected to follow a square-root law [24]. By using four $\mathrm{Al}$ absorbers of 10, 13, 14.6 and $17.3 \mu \mathrm{m}$ thicknesses, in front of the ${ }^{241} \mathrm{Am}$ alpha source, we scaled the incident energy from $5.48 \mathrm{MeV}$ to $3.76,3.18,2.72$ and $2.19 \mathrm{MeV}$ respectively as measured by the calibrated silicon detector (see Fig. 3). Using the SRIM

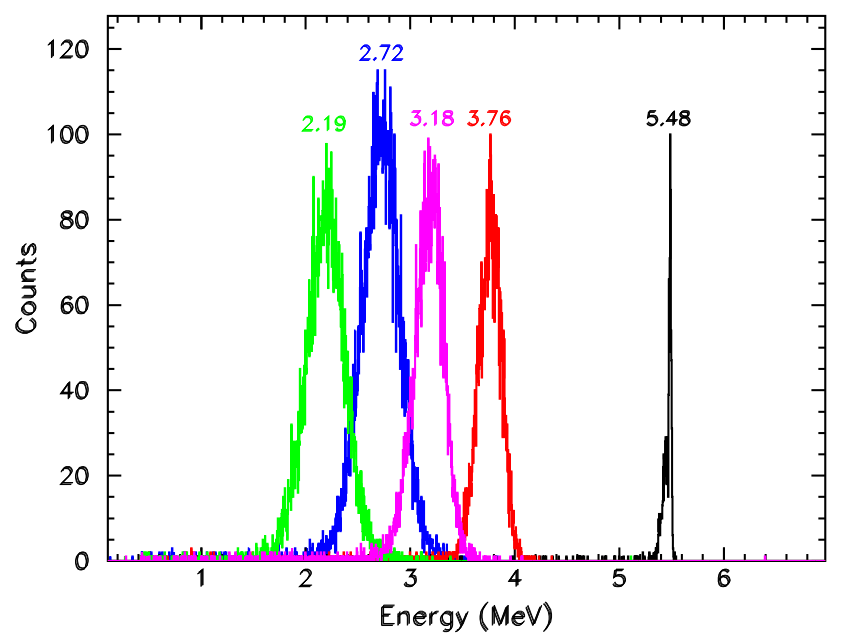

Fig. 3. The four ${ }^{241} \mathrm{Am}$ alpha energy spectra, measured by the Si detector with the $10,13,14.6$ and $17.3 \mu \mathrm{m} \mathrm{Al}$ absorbers in front of the source. The $5.48 \mathrm{MeV}$ peak is also shown for reference.

code [25] calculations, the ranges of the alpha particles at the four incident energies in the $\mathrm{SiC}$ material were evaluated to be: $10.8 \pm 0.4,8.5 \pm 0.5,7.0 \pm 0.6$ and $5.4 \pm 0.5 \mu \mathrm{m}$ respectively. The errors on the range thickness were evaluated by taking into account both the energy loss in the $\mathrm{Ni}_{2} \mathrm{Si} 0.2 \mu \mathrm{m}$ thick front layer on the detector and the FWHM (full-width at half-maximum) of the alpha spectra of Fig. 3, in order to account for the energy straggling introduced by the $\mathrm{Al}$ absorbers. A typical set of spectra is shown in Fig. प for one of the detectors used. For all the detectors the centroid position of the peak moves toward higher channels as the voltage increases. The observed shift can be described according to the increasing thickness of the active volume of the 
detector with increasing reverse bias voltage. As a result, the incident alphas
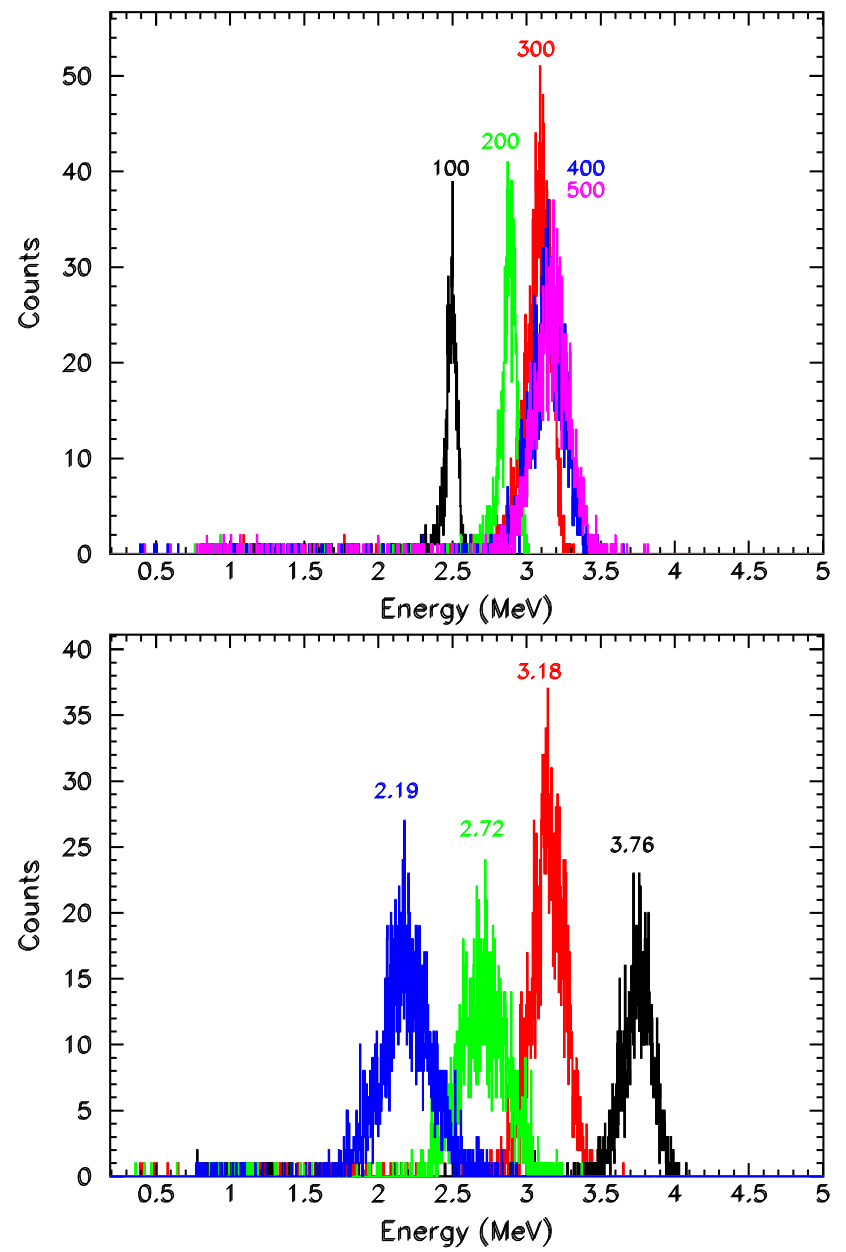

Fig. 4. Top: Energy spectra measured by the $\mathrm{SiC}$ diode at increasing bias voltage for alpha particles of $3.18 \mathrm{MeV}$ incident energy. Bottom: The four alpha energy spectra $(2.19,2.72,3.18,3.76 \mathrm{MeV})$ measured by the $\mathrm{SiC}$ diode at the bias saturation values.

deposit more energy in the active region leading to detector signals with higher pulse heights. The saturation of the pulse height values is then reached when the applied reverse voltage depletes the active volume of the diode up to the range corresponding to the energy of the incoming alpha. For a given alpha energy we then searched for the value of the applied reverse bias at which the saturation of the pulse height signals is reached (see Fig. 4-top panel). The correlation between the evaluated range and the applied reverse voltage, 
shown in Fig. 5, is nicely reproduced by a square-root law [24].

Finally, by correlating the saturated pulse height peak values and the four

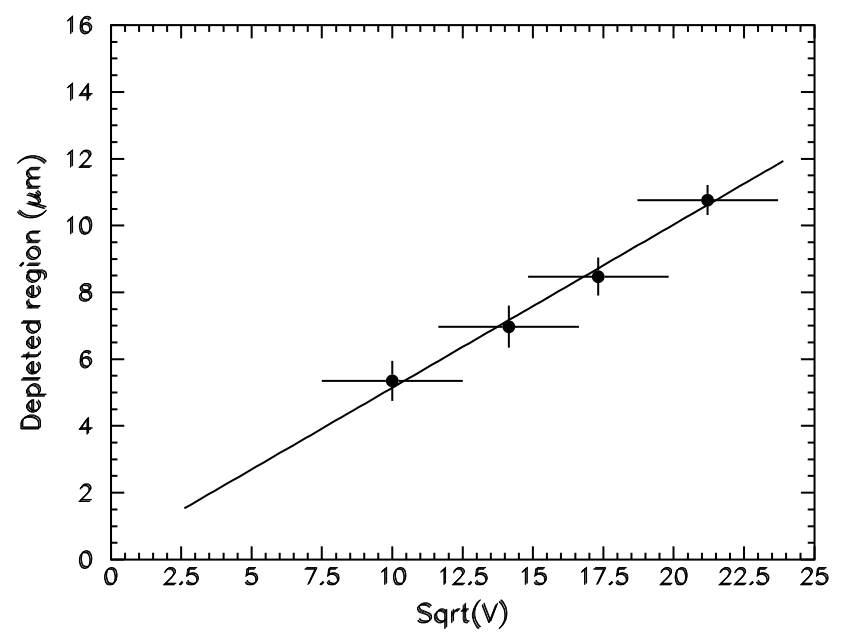

Fig. 5. Correlation between the square root of the applied bias and the SiC depletion layer thickness evaluated from alpha energies.

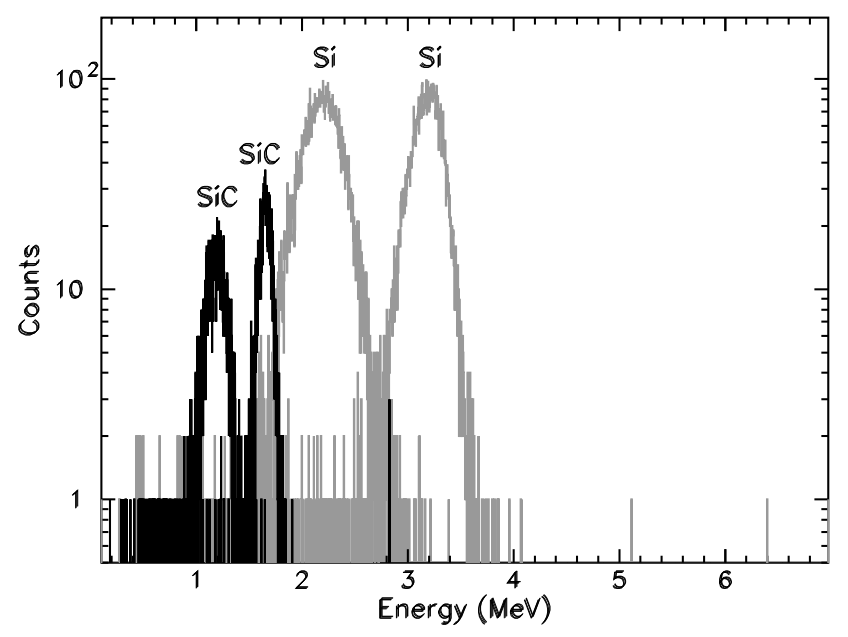

Fig. 6. Energy spectra of alpha particles of 2.19 and $3.18 \mathrm{MeV}$ incident energy measured in the silicon (gray) and in the $\mathrm{SiC}$ (black) detectors. For comparison the energy values of the SiC spectra were evaluated by using the energy calibration obtained for the silicon detector.

alpha energies $(2.08,2.62,3.09,3.68 \mathrm{MeV})$ corrected for the energy lost in the $\mathrm{Ni}_{2} \mathrm{Si} 0.2 \mu \mathrm{m}$ thick front layer of the $\mathrm{SiC}$ diode, we calibrated the pulse height scale. By comparing the energy peaks obtained with the $\mathrm{SiC}$ and the 
silicon detector for the same deposited energy (see Fig. 6) we have obtained a factor of $2.1 \pm 0.3$ in the charge produced by ionization, as expected from the different ionization energy values of $3.76 \mathrm{eV}$ in $\mathrm{Si}$ and of $7.74 \mathrm{eV}$, as recently measured [26]27], in $\mathrm{SiC}$.

To increase the explored energy range we used beams of ${ }^{12} \mathrm{C}$ at 5.06 and 17.68 $\mathrm{MeV}$ and ${ }^{16} \mathrm{O}$ at $7.3,9.78,12.18$ and $14.21 \mathrm{MeV}$ of incident energies provided by the Tandem accelerator of the LNS. The SiC diodes were operated at a reverse bias of $-100 \mathrm{~V}$ which depletes the active region of the detector up to $5.4 \pm 0.5 \mu \mathrm{m}$, wide enough to completely stop all the ions except the ${ }^{12} \mathrm{C}$ at 17.68 $\mathrm{MeV}$ and the ${ }^{16} \mathrm{O}$ at $14.21 \mathrm{MeV}$ of incident energy which, according to SRIM calculation, release around 8 and $13 \mathrm{MeV}$ respectively in the detector. Fig. 7 shows the energy spectra measured by the SiC diodes. The high degree of linearity observed in the correlation between the pulse height and the energy shown in Fig. 8 for $\mathrm{SiC}$ detectors, is consistent with previous measurements [13, 14, 19], and indicates the proportionality of the produced ionization charge to the deposited energy. Similarly to the measurements performed with the alpha source, a number of spectra were taken over a range of bias voltages, from 0 to $-450 \mathrm{~V}$, for the ${ }^{12} \mathrm{C}$ beam at $17.68 \mathrm{MeV}$ incident energy ( see Fig. 7 . bottom panel). We notice in Fig. (7) that no saturation has been observed in the peak centroid values, since the active region of the detector does not extend beyond the range of the ${ }^{12} \mathrm{C}$ at $17.68 \mathrm{MeV}$ in $\mathrm{SiC}$, which is approximately $11.2 \mu \mathrm{m}$. This occurrence indicates that at the maximum applied bias voltage, - $450 \mathrm{~V}$, the detector is only about half depleted but, unfortunately, we could not raise the bias voltage to higher values because the electrical connections in vacuum were sparking at around $-500 \mathrm{~V}$. Therefore we evaluated the thickness of the depleted region by comparing SRIM calculations to the energy loss measured at different applied biases. Figure 9 shows the correlation between 

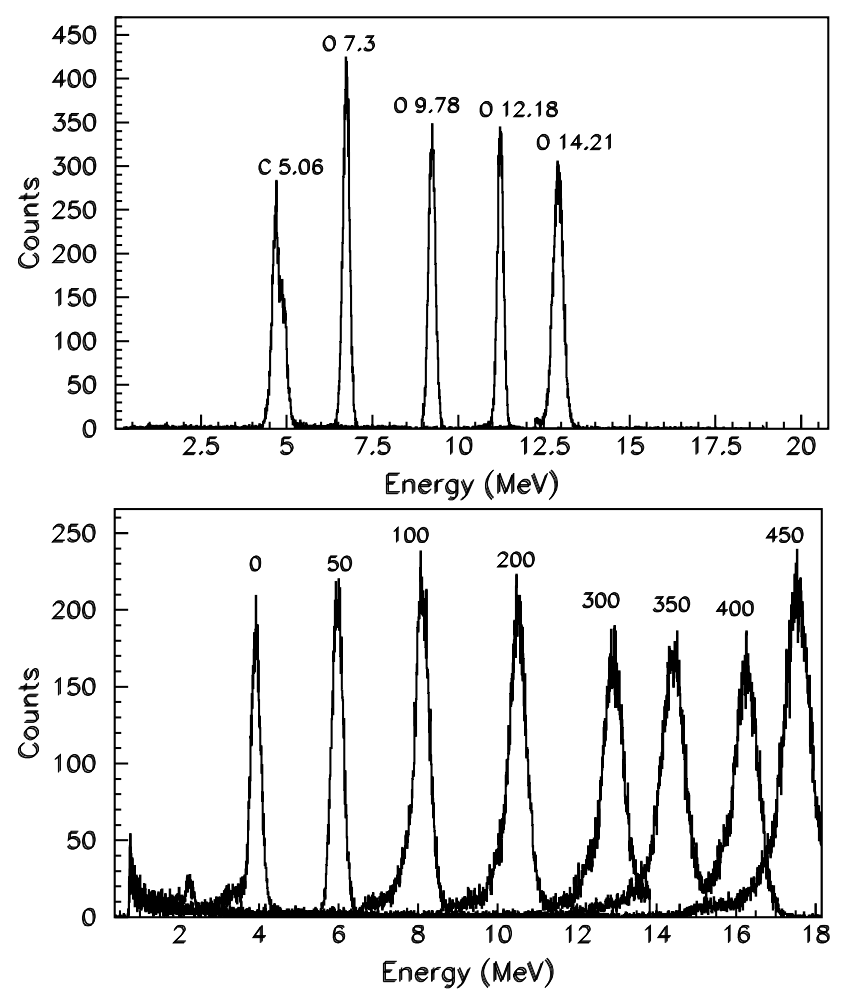

Fig. 7. Top: $\mathrm{SiC}$ energy spectra of ${ }^{12} \mathrm{C}$ and ${ }^{16} \mathrm{O}$ beams at different energies. Bottom: $17.68 \mathrm{MeV}{ }^{12} \mathrm{C}$ beam spectra measured at increasing negative bias voltages.

the estimated thickness of the depletion layer and the applied reverse bias for ${ }^{12} \mathrm{C}$ ions. We remark the good agreement with the data measured with alphas and the good linear correlation between the depletion layer thickness and the square-root of the applied bias values. From a linear fit (see Fig. 9), at zero bias voltage we estimated an active region thickness of $1.18 \mu \mathrm{m}$ resulting only from the Schottky contact potential, in good agreement with the result of Ref. [13].

In order to measure how the energy resolution behaves as a function of the thickness of the active region and therefore of the applied bias voltage, we analyzed the data reported in Fig. 7. A typical ${ }^{12} \mathrm{C} 17.68 \mathrm{MeV}$ response spectrum is shown in Fig. 10: the shape of the peak is well reproduced by a Gaussian function. Based on the energy calibration, the full-width at half-maximum 


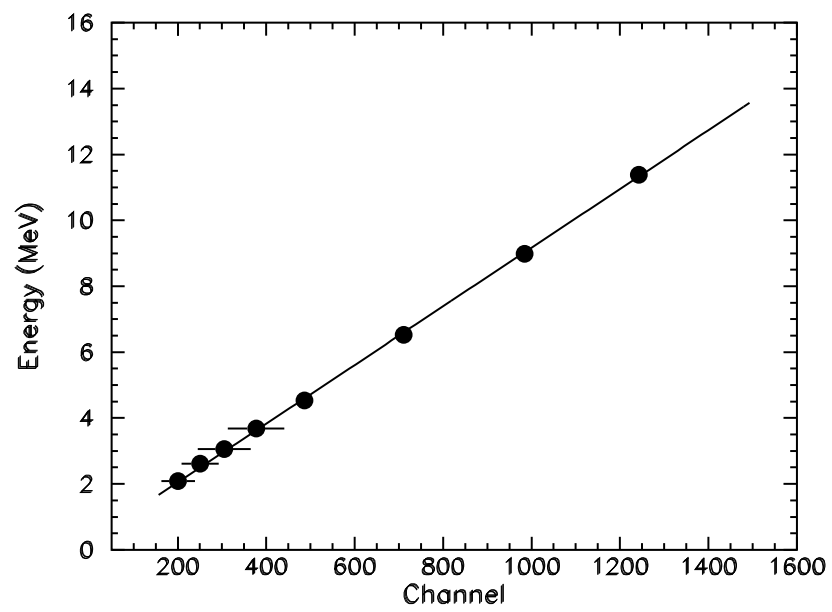

Fig. 8. Pulse height(channel) versus Energy plot. Data points refer to the ${ }^{12} \mathrm{C}(5.06$ (4.5)MeV) and ${ }^{16} \mathrm{O}(7.3(6.5), 9.78(9.0), 12.18(11.4) \mathrm{MeV})$ beams and to the four alpha particles energies. The energy values in parentheses are the ones corrected for the energy lost in the $\mathrm{Ni}_{2} \mathrm{Si} 0.2 \mu \mathrm{m}$ thick front layer on the detector. The linear correlation coefficient $\mathrm{R}^{2}$ from the fit has a value of 0.998 .

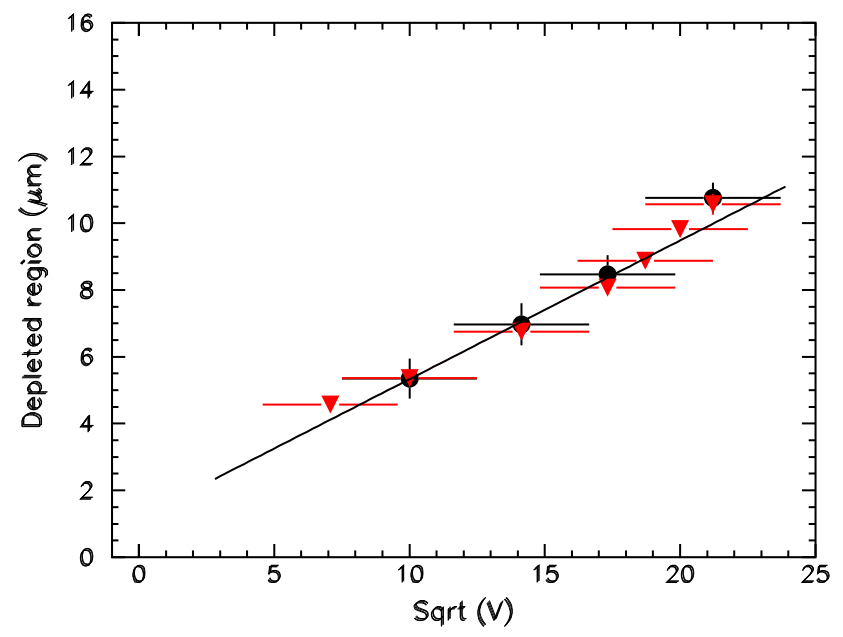

Fig. 9. Correlation between the square root of the applied bias and the SiC depletion layer thickness evaluated from alpha (circles) and the $17.68 \mathrm{MeV}{ }^{12} \mathrm{C}$ beam (triangles) spectra. Error bars are calculated from the FWHM of the energy loss spectra of Fig. 7 ,

(FWHM) of the Gaussian fit to the ${ }^{12} \mathrm{C}$ peak at $8.1 \mathrm{MeV}$ (which is the energy lost in $5.4 \mu \mathrm{m}$ by the ${ }^{12} \mathrm{C}$ at $17.68 \mathrm{MeV}$ ) is $190 \mathrm{keV}$ corresponding to an energy 

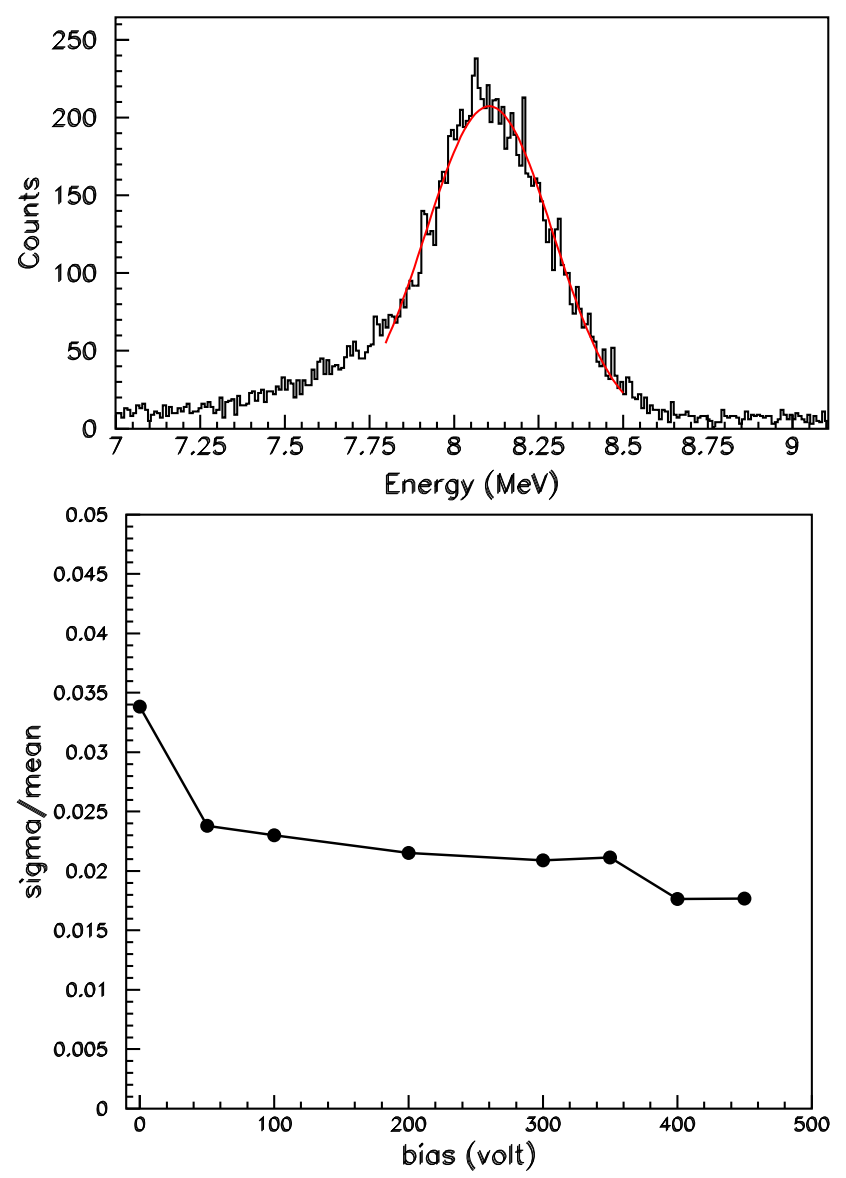

Fig. 10. Top: ${ }^{12} \mathrm{C}$ at $17.68 \mathrm{MeV}$ beam response of a $\mathrm{SiC}$ diode with an applied bias voltage of -100 Volts. The spectrum is fitted with a Gaussian function. Bottom: Relative energy resolution as a function of the applied bias voltage.

resolution of $2.3 \%$. In the bottom panel of Fig. 10, the relative energy resolution (FWHM of the peak over the peak position), is shown as a function of the applied bias voltage. Besides an initial enhancement from 0 to $-50 \mathrm{~V}$, the relative resolution decreases slowly over the range of the applied bias voltages up to a value of $1.8 \%$. The measured energy resolution values are in good agreement with the ones observed in other measurements [14] although larger than the recently reported $\mathrm{SiC}$ energy resolution value of $0.34 \%$ [27, 28].

Moreover by storing the waveforms of the preamplifier output in the Tektronix TDS 5104B Digital Oscilloscope we analyzed the behavior of the pulses gener- 


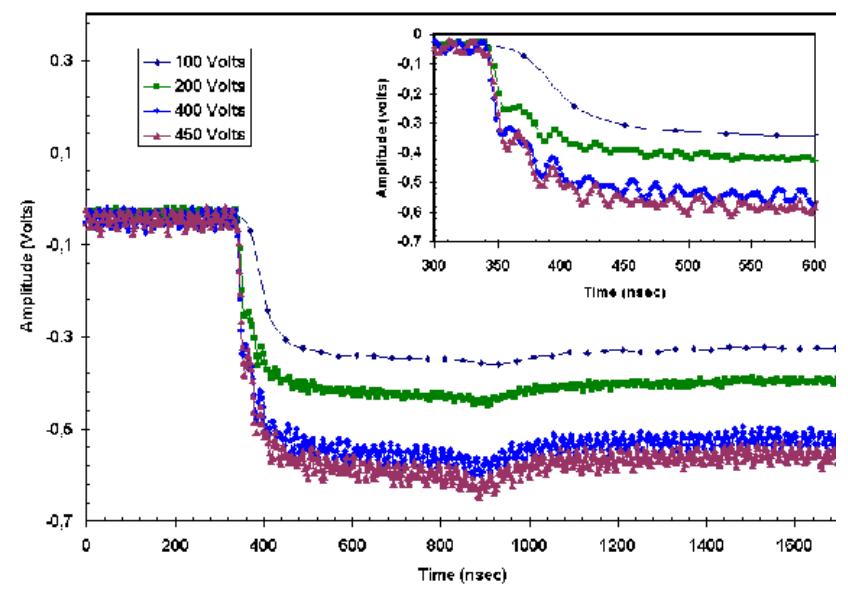

Fig. 11. Signals from the preamplifier generated by ${ }^{12} \mathrm{C}$ ions at $17.68 \mathrm{MeV}$ incident energy for different applied bias values.

ated by the ${ }^{12} \mathrm{C}$ beam at $17.68 \mathrm{MeV}$. Typical pulses are reported in Fig. 11] for four different reverse bias values. The amplitude variation is obviously related to the increasing thickness of the active region and therefore to the increasing energy lost by the ${ }^{12} \mathrm{C}$ ions. The decrease of the signal rise-time is related to the combined variations of the drift velocity of the ionization charges, proportional to $\mathrm{V}_{\text {Bias }}$, and of the depleted region thickness, proportional to $\sqrt{V_{\text {Bias }}}$. Indeed, the rise-time varies from 97 nsec at -100 Volts to 68 nsec at -200 Volts and reaches 46 and 44 nsec at -400 and -450 Volts respectively. As expected the ratios of the measured rise-time values scale as the inverse ratio of the square-root of the applied bias voltages.

The consistency of the $\mathrm{SiC}$ detectors performances were investigated by testing charged-particle response of all the eleven detectors of identical configuration. All of them showed, within the error bars, the same characteristics here reported. 


\section{Radiation damage}

One of the main motivations of such study was to explore the radiation hardness properties of such $\mathrm{SiC}$ detectors. Five samples were irradiated using $53 \mathrm{MeV}{ }^{16} \mathrm{O}$ ions provided by the Tandem at the LNS-Catania and their output signals were monitored during the irradiation time. Fig. 12 shows the peak centroid of the ${ }^{16} \mathrm{O}$ spectrum as a function of the fluence. The applied

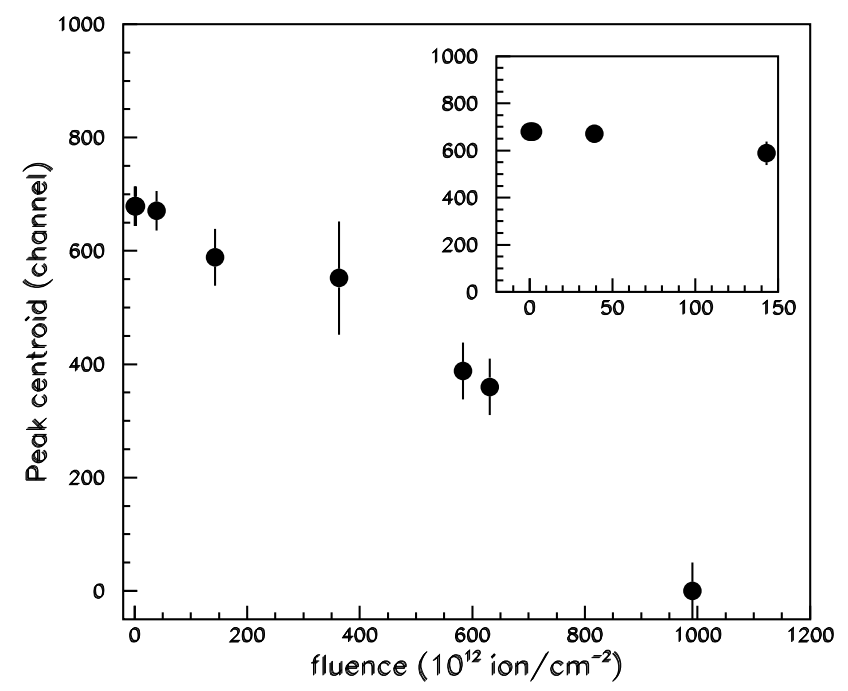

Fig. 12. Peak value of the spectrum of $53 \mathrm{MeV}{ }^{16} \mathrm{O}$ beam measured at increasing fluence. The plot at the lowest values of fluence is expanded in the insert.

reverse bias was kept fix at $-100 \mathrm{~V}$ but the signals appeared to decrease in amplitude as the irradiation increased. The amplitude dropped to $50 \%$ at a fluence of $6.510^{14}$ ions $/ \mathrm{cm}^{2}$ (see Fig. 12) in good agreement with results of Ref. 20]29]. At the same time, the reverse current increased by a factor of five and the noise by a factor of two (see Fig. 13). The present $\mathrm{SiC}$ diodes are therefore around ten times radiation harder than silicon diodes but around a factor ten less hard than thin epitaxial Si-diodes for which the charge collection efficiency drops only to about $80 \%$ at $610^{15}$ protons $/ \mathrm{cm}^{2}$ at $24 \mathrm{GeV}$ of incident energy [30]. 


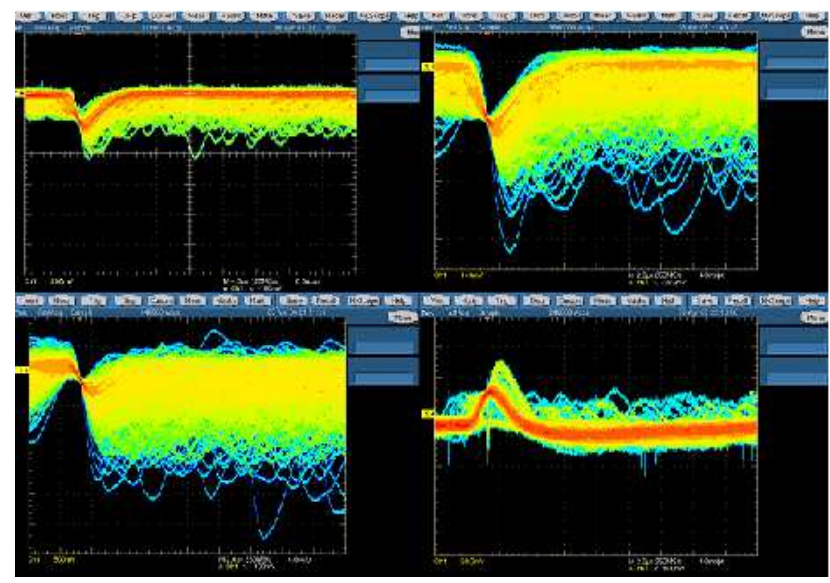

Fig. 13. Pulses from a $\mathrm{SiC}$ diode irradiated with the $53 \mathrm{MeV}{ }^{16} \mathrm{O}$ beam. Top left: beginning of irradiation. Top right: after a fluence of $3.510^{14} \mathrm{ions} / \mathrm{cm}^{2}$. Bottom left: after a fluence of $710^{14}$ ions $/ \mathrm{cm}^{2}$. Bottom right: after a fluence of $10^{15}$ ions $/ \mathrm{cm}^{2}$ : notice the inversion of the signal.

However it is not clear how to compare the damage produced by low energetic ${ }^{12} \mathrm{C}$ or ${ }^{16} \mathrm{O}$ ions to the damage produced by protons, neutrons, gammas or pions and which kind of defects, among the known ones, are produced during irradiation.

Finally, two different detectors were continuously observed during the irradiation up to when they break-down at a fluence of $10^{15}$ ions $/ \mathrm{cm}^{2}$. At this irradiation fluence we observed an inversion of the signals with respect to the non-irradiated ones (see Fig. 13, bottom-right panel). This effect seems similar to the symmetric Charge Collection Efficiency (CCE) response at both polarities mentioned in Ref. [20] at a fluence of $10^{15}$ protons $/ \mathrm{cm}^{2}$ and attributed to radiation damage.

Moreover, we inverted both bias and amplifier polarities and we sent on the irradiated detectors an ${ }^{16} \mathrm{O}$ beam at $12 \mathrm{MeV}$ incident energy. Since the applied reverse bias of $-100 \mathrm{~V}$ depletes $5.4 \mu \mathrm{m}$ of the $\mathrm{SiC}$ active region, we expected an energy release of 8.3 and $12 \mathrm{MeV}$ in the detector from the ${ }^{16} \mathrm{O}$ beam at 53 and 
$12 \mathrm{MeV}$ incident energy respectively, but the pulse height of the signal was the same in both cases. Therefore we conclude that the detector was sensitive to the charged ion but not to the deposited energy.

Further analysis will be performed to understand if the observed effect is of the same nature of space-charge sign-inversion ("type inversion"), reported for Si-diodes [31/32,33]. At the moment we suggest, as possible explanation, the formation of a layer of ${ }^{16} \mathrm{O}\left(10^{15} \mathrm{ions} / \mathrm{cm}^{2}\right)$ at a distance of $27.2 \pm 0.4 \mu \mathrm{m}$ (which is the range of the $53 \mathrm{MeV}^{16} \mathrm{O}$ ions in the $\mathrm{SiC}$ material) from the Schottky contact, and therefore in the $\mathrm{n}^{+}$type substrate, subdividing the $300 \mu \mathrm{m}$ thick material in two parts with a floating ground at the ${ }^{16} \mathrm{O}$ layer.

\section{Conclusions}

In the present work we investigated the response of $\mathrm{SiC}$ Schottky diodes to alphas, ${ }^{12} \mathrm{C}$ and ${ }^{16} \mathrm{O}$ low energetic ions in order to explore the possibility of using these detectors in nuclear physics applications in extreme environments. The signal response to the ionization produced by the low-energy ions was analyzed in terms of linearity, energy resolution, rise-time and deterioration as function of the applied reverse bias and the irradiating fluence. The latter measurements demonstrate the good quality of the $\mathrm{SiC}$ as a radiation hard material.

Energy resolution on the order of $3 \%$ and rise time up to 44 nsec have been measured at the maximum applied reverse bias. The amplitude of the signal drops down to $50 \%$ at a fluence of $6.510^{14}$ ions $/ \mathrm{cm}^{2}$ indicating more than an order of magnitude hardness to the radiation with respect to silicon diodes. The signal inversion at a fluence of $10^{15}$ ions $/ \mathrm{cm}^{2}$ should be further inves- 
tigated to understand if it is produced by radiation damage or by the ${ }^{16} \mathrm{O}$ implant in the material.

The main inconvenience we have found in the use of the present detectors was the high bias voltage, needed to deplete the active region of the diode. We expect a correlation between the depleting bias values and the $\mathrm{N}$ doping concentration in the epitaxial region [13]. We will investigate in the near future such a correlation, being aware of the negative influence on the ionization charge production and radiation hardness of a reduced doping concentration. But reduction of the applied voltage to values far from the break down ones is a very crucial issue for future applications.

\section{Acknowledgements}

The help in the $\mathrm{SiC}$ diodes selection and assembling and the valuable discussion with L.Calcagno, G.Foti and F.La Via are greatly appreciated. E.R. acknowledges the support from the European Community in the framework of the "DIRAC secondary-beams" -contract N.515873 under the "Structuring the European Research Area" Specific Programme Research Infrastructures action.

\section{References}

[1] R.J. Trew, J.-B. Yan, P.M. Mock, Proc. IEEE 79 (1991) 598.

[2] B.J. Baliga, Proc. IEEE 82 (1994) 1112.

[3] C.E. Weitzel, C.E. Palmour, C.H. Carter Jr., K. Moore, K.J. Nordquist, S. Allen, C. Thero, M. Bhatnagar, IEEE Trans. Electron Dev. 43 (1996) 1732. 
[4] G.T. Heydt, B.J. Skromme, Applications of high power electronic switches in the electric power utility industry and the needs for high power switching devices, in: S.J. Pearton, R.J. Shul, E. Wolf gang, F. Ren, S. Tenconi (Eds.), Power Semiconductor Materials and Devices, Materials Research Society Symposia Proceedings, Vol.483, Materials Research Society, Warrandale, PA, 1998, p.3.

[5] IEEE Trans. Electron Devices 46 (3) (1999) (number dedicated to silicon carbide).

[6] J.A. Cooper, A. Agarwal, Proc. IEEE 90 (6) (2002) 956.

[7] A. Elasser, T.P. Chow, Proc. IEEE 90 (6) (2002) 969.

[8] R.C. Clarke, J.W. Palmour, Proc. IEEE 90 (6) (2002) 987.

[9] M. Rogalla, K. Runge, A. Soldner-Rembold, Nucl. Phys. B 78 (Proc. Suppl.) (1999) 516.

[10] M. Bruzzi, F. Nava, S. Russo, S. Sciortino, P. Vanni, Diam. Relat. Mater. 10 (2001) 657.

[11] A.R. Dulloo, F.H. Ruddy, J.G. Seidel, J.M. Adams, J.S. Nico, D.M. Gilliam, Nucl. Instr. and Meth. A 422 (1999) 47.

[12] G. Bertuccio, R. Casiraghi, A. Cetronio, C. Lanzieri, F. Nava, Nucl. Instr. and Meth. A 522 (2004) 413.

[13] F. Nava, P. Vanni, C. Lanzieri, C. Canali, Nucl. Instr. and Meth. A 437 (1999) 354.

[14] F.H. Ruddy, A.R. Dulloo, J.G. Seidel, J.W. Palmour, R. Singh, Nucl. Instr. Meth. A 505 (2003) 159

[15] A. Kinoshita, M. Iwami, K. Kobayashi, I. Nakano, R. Tanaka, T. Kamiya, A. Ohi, T. Ohshima, Y. Fukushima, Nucl. Instr. Meth. A 541 (2005) 213

[16] W. Cunningham, A. Gouldwell, G. Lamb, J. Scott, K. Mathieson, P. Roy, R. Bates, P. Thornton, K.M. Smith, R. Cusco, M. Glaser, M. Rahman, Nucl. Instr. 
and Meth. A 487 (2002) 33

[17] F. Nava, E. Vittone, P. Vanni, G. Verzellesi, P.G. Fuochi, C. Lanzieri, M. Glaser, Nucl. Instr. and Meth. A 505 (2003) 645.

[18] W. Cunningham, J. Melone, M. Horn, V. Kazukauskas, P. Roya, F. Doherty, M. Glaser, J. Vaitkus, M. Rahman, Nucl. Instr. and Meth. A 509 (2004) 127.

[19] W. Cunningham, J. Melone, M. Horn, V. Kazukauskas, P. Roy, F. Doherty, M. Glaser, J. Vaitkus, M. Rahman, Nucl. Instr. and Meth. A 509 (2003) 127.

[20] S. Sciortino, F. Hartjes, S. Lagomarsino, F. Nava, M. Brianzi, V. Cindro, C. Lanzieri, M. Moll, P. Vanni, Nucl. Instr. and Meth. A 552 (2005) 138

[21] K.K. Lee, T. Ohshima, A. Saint, T. Kamiya, D.N. Jamieson, H. Itoh, Nucl. Instr. and Meth. B 210 (2003) 489.

[22] E.T.C. Epitaxial Tecnology Center 207, Corso Italia I-95127 Catania -Italy

[23] F. La Via, F. Roccaforte, A. Maktari, V. Raineri , P. Musumeci, L. Calcagno, Microelectronic Engineering, Vol.60 (2002) 269

F. Roccaforte, F. La Via, V. Raineri, P. Musumeci and L. Calcagno, Appl. Phys. A (2003) 827

[24] G.F. Knoll, Radiation Detection and Measurement, Third Edition, Wiley, New York, 2000, p.393

[25] J.F. Ziegler, J.P. Biersack, IBM-research, Yorktown Heights, New York, USA, 1996.

[26] G. Bertuccio and R. Casiraghi, IEEE Trans. Nuc. Sci. 50 (2003) 175

[27] A. Ivanov, E. Kalinina, G. Kholuyanov, N. Stroka, G. Onushkin, A. Konstantinov, A. Hallen, A. Kuznetsov, in Silicon Carbide and Related Materials 2004, R. Nipoti, et al. (Eds.), Materials Science Forum 483-484, pp $1029-1032$

[28] F.H. Ruddy, J.G. Seidel, H.Chen, A.R. Dulloo,S. Ryu, IEEE Trans. Nucl. Sci. $53[2006] 1713$ 
[29] T. Quinn, R. Bates, M. Bruzzi, W. Cunningham, K. Mathieson, M. Moll, T. Nelson, H.E. Nilsson, I. Pintillie, L. Reynolds, S. Sciortino, P. Sellin, H. Strachan, B.G. Svensson, J. Vaitkus, M. Rahman, IEEE Nuclear Science Symposium Portland, OR, USA, 19-25 Oct. 2003. Conference Record, Vol.2, p.1028-33, 2004. Ed. by S.D. Metzler, 2003

[30] G. Lindström, E. Fretwurst, F. Honniger, G. Kramberger, M. Moller-Ivens, I. Pintilie, A. Schramm, Nucl. Instr. and Meth. A 556 (2006) 451

[31] G. Lindström et al., "ROSE Collaboration", Nucl. Instr. and Meth. A 466 (2001) 308.

[32] I.E. Anokhin, A.B. Rosenfeld, O.S. Zinets, Radiation Protection Dosimetry 101 (2002) 107-110.

[33] L. Bosisio, S. Dittongo, E. Quai and I. Rachevskaia, IEEE Trans. Nucl. Sci., 50 (1) (2003) 219 